\title{
On Existence of Multiplicity of Weak Solutions for a New Class of Nonlinear Fractional Boundary Value Systems via Variational Approach
}

\author{
Fares Kamache, ${ }^{1}$ Salah Mahmoud Boulaaras $\mathbb{D}^{2,3}$ Rafik Guefaifia, ${ }^{1}$ Nguyen Thanh Chung, \\ Bahri Belkacem Cherif $\left(\mathbb{D},{ }^{2,5}\right.$ and Mohamed Abdalla ${ }^{6,7}$ \\ ${ }^{1}$ Laboratory of Mathematics, Informatics and Systems, Larbi Tebessi University, 12000 Tebessa, Algeria \\ ${ }^{2}$ Department of Mathematics, College of Sciences and Arts, ArRass, Qassim University, Saudi Arabia \\ ${ }^{3}$ Laboratory of Fundamental and Applied Mathematics of Oran (LMFAO), University of Oran 1, Oran, 31000 Oran, Algeria \\ ${ }^{4}$ Department of Mathematics, Quang Binh University, 312 Ly Thuong Kiet, Dong Hoi, Quang Binh, Vietnam \\ ${ }^{5}$ Preparatory Institute for Engineering Studies, Sfax, Tunisia \\ ${ }^{6}$ Mathematics Department, College of Science, King Khalid University, Abha 61413, Saudi Arabia \\ ${ }^{7}$ Mathematics Department, Faculty of Science, South Valley University, Qena 83523, Egypt \\ Correspondence should be addressed to Bahri Belkacem Cherif; bahi1968@yahoo.com
}

Received 10 February 2021; Revised 1 March 2021; Accepted 5 March 2021; Published 13 March 2021

Academic Editor: Kamyar Hosseini

Copyright ( 92021 Fares Kamache et al. This is an open access article distributed under the Creative Commons Attribution License, which permits unrestricted use, distribution, and reproduction in any medium, provided the original work is properly cited.

This paper deals with the existence of solutions for a new class of nonlinear fractional boundary value systems involving the left and right Riemann-Liouville fractional derivatives. More precisely, we establish the existence of at least three weak solutions for the problem using variational methods combined with the critical point theorem due to Bonano and Marano. In addition, some examples in $\mathbb{R}^{3}$ and $\mathbb{R}^{4}$ are given to illustrate the theoritical results.

\section{Introduction}

Fractional differential equations (FDEs) are a generalization of ordinary differential equations (ODEs), as they contain fractional derivatives whose degree is not necessarily an integer. This is what makes it receive great attention from researchers due to its ability to model some difficult and complex phenomena in many fields, including engineering, science, biology, economics, and physics (for more information, see [1-22]). One of the most investigated issues is the existence of solutions for the fractional initial and boundary value problems by using some fixed point theorems, coincidence degree theory, and monotone interactive method. Among the most important of these are the works mentioned in Oldham and Spanier and Podlubny's books (see $[13,23]$ ) and the work of Metzler and Klafter (see [24]). Furthermore, the first to use the critical point theorem was Jiao and Zhou in [6] to study the following problem:

$$
\left\{\begin{aligned}
{ }_{t} D_{T}^{\alpha}\left({ }_{0} D_{t}^{\alpha} u(t)\right) & =\nabla F(t, u(t)), \quad \text { a.e } t \in[0, T], \\
u(0)=u(t) & =0,
\end{aligned}\right.
$$

where ${ }_{0} D_{T}^{\alpha}$ and ${ }_{t} D_{T}^{\alpha}$ are the left and right Riemann-Liouville fractional derivatives with $0<\alpha \leq 1$, respectively, and $F$ : [0, $T] \times \mathbb{R} \longrightarrow \mathbb{R}^{n}$ is a suitable function satisfying some hypothesis and $F(t, x)$ is the gradient of $F$ with respect to $x$.

In [22], the authors have used variational methods to investigate the existence of weak solutions for the following system: 


$$
\left\{\begin{array}{cc}
{ }_{t} D_{T}^{\alpha}\left(a(t){ }_{0} D_{t}^{\alpha} u(t)\right)=\lambda F_{u}(t, u(t), v(t)), & \text { a.e } t \in[0, T], \\
{ }_{t} D_{T}^{\beta}\left(b(t){ }_{0} D_{t}^{\beta} v(t)\right)=\lambda F_{v}(t, u(t), v(t)), & \text { a.e } t \in[0, T], \\
u(0)=u(T)=0, v(0)=v(T)=0, &
\end{array}\right.
$$

for ${ }_{0} D_{T}^{\alpha}$ and ${ }_{t} D_{T}^{\alpha}$ are the left and right Riemann-Liouville fractional derivatives with $0<\alpha \leq 1$ and $F_{s}$ denotes the par- tial derivative of $F$ with respect to $s$. In [?], Zhao et al. obtained the existence of infinitely many solutions for system (2) with perturbed functions $h_{i}, i=1,2$.

Yet, there are a few findings for fractional boundary value problems which were established exploiting this approach due to its difficulty in establishing a suitable space and variational functional for fractional problems.

In this work, we shall study the existence of three weak solutions for the following system:

$$
\left\{\begin{array}{l}
{ }_{t} D_{T}^{\alpha_{i}}\left(a_{i}(t){ }_{0} D_{t}^{\alpha_{i}} u_{i}(t)\right)=\lambda F_{u_{i}}\left(t, u_{1}(t), u_{2}(t), \cdots, u_{n}(t)\right)+h_{i}\left(u_{i}(t)\right), \quad \text { a.e } t \in[0, T], \\
\quad u_{i}(0)=u_{i}(T)=0
\end{array}\right.
$$

for $1 \leq i \leq n$, where $\alpha_{i} \in(0 ; 1],{ }_{0} D_{T}^{\alpha_{i}}$ and ${ }_{t} D_{T}^{\alpha_{i}}$ are the left and right Riemann-Liouville fractional derivatives of order $\alpha_{i}$, respectively, $a_{i} \in L^{\infty}([0, T])$ with

$$
a_{i 0}=\text { ess } \inf _{[0, T]} a_{i}>0, \quad \text { for } 1 \leq i \leq n,
$$

$\lambda>0, F:[0, T] \times \mathbb{R}^{n} \longrightarrow \mathbb{R}$ is a measurable function for all $\left(x_{1}, \cdots, x_{n}\right) \in \mathbb{R}^{n}$ and is $C^{1}$ with respect to $\left(x_{1}, \cdots, x_{n}\right) \in$ $\mathbb{R}^{n}$ for a.e. $t \in[0, T], F_{u_{i}}$ denotes the partial derivative of $F$ with respect to $u_{i}$, respectively, and $h_{i}: \mathbb{R} \longrightarrow \mathbb{R}$ are Lipschitz continuous functions with the Lipschitz constants $L_{i}>0$, for $1 \leq i \leq n$, i.e.,

$$
\left|h_{i}\left(x_{1}\right)-h_{i}\left(x_{2}\right)\right| \leq L_{i}\left|x_{1}-x_{2}\right|
$$

for all $x_{1}, x_{2} \in \mathbb{R}$ and $h_{i}(0)=0$, for $1 \leq i \leq n$. In order to state the main results, we introduce the following conditions:

(F0) For all $C>0$ and any $1 \leq i \leq n$

$$
\sup _{\left|\left(x_{1}, \cdots, x_{n}\right)\right| \leq C}\left|F_{u_{i}}\left(t, x_{1}, \cdots, x_{n}\right)\right| \in L^{1}([0, T]) .
$$

(F1) $F(t ; 0, \cdots, 0)=0$, for a.e. $t \in[0 ; T]$.

In the present study, motivated by the results introduced in $[12,13,25]$, using the three critical point theorems due to Ricceri ([26], see Theorem 2.6 in the next section), we ensure the existence of at least three solutions for system (3). For other applications of Ricceri's result for perturbed boundary value problems, the interested readers are referred to the papers [11-13, 23-25, 27].

We divided the paper as follows: in the second section, we put some preliminary facts, while in the third section we presented the main result and its proof. Finally, we proposed two practical examples of our theorem.

\section{Preliminaries}

In this section, introducing some necessary definitions and preliminary facts.
Definition 1 [28]. Let $u$ be a function defined on $[0, T]$ and $\alpha_{i}>0$ for $1 \leq i \leq n$. The left and right Riemann-Liouville fractional integrals of order $\alpha_{i}$ for the function $u$ are defined by

$$
\begin{array}{ll}
{ }_{0} D_{t}^{-\alpha_{i}} u(t)=\frac{1}{\Gamma\left(\alpha_{i}\right)} \int_{0}^{t}(t-s)^{\alpha_{i}-1} u(s) d s, & t \in[0, T], \\
{ }_{0} D_{t}^{-\alpha_{i}} u(t)=\frac{1}{\Gamma\left(\alpha_{i}\right)} \int_{t}^{T}(s-t)^{\alpha_{i}-1} u(s) d s, & t \in[0, T],
\end{array}
$$

for $1 \leq i \leq n$, provided the RHS are pointwise given on $[0, T]$, where $\Gamma\left(\alpha_{i}\right)$ is the standard gamma function defined by

$$
\Gamma(z)=\int_{0}^{+\infty} z^{\alpha_{i}-1} e^{-z} d z
$$

Definition 2 [25]. Let $0<\alpha_{i} \leq 1$ for $1 \leq i \leq n$. The fractional derivative space $H_{0}^{\alpha_{i}}$ is given by the closure $C_{0}^{\infty}([0, T], \mathbb{R})$, that is

$$
H_{0}^{\alpha_{i}}=C_{0}^{\infty}([0, T], \mathbb{R}),
$$

with the norm

$$
\left\|u_{i}\right\|_{\alpha_{i}}=\left(\int_{0}^{T} a_{i}(t)\left|{ }_{0} D_{t}^{\alpha_{i}} u_{i}(t)\right|^{2} d t+\int_{0}^{T}\left|u_{i}(t)\right|^{2} d t\right)^{1 / 2}
$$

for every $u_{i} \in H_{0}^{\alpha_{i}}$ and for $1 \leq i \leq n$.

We point out that $H_{0}^{\alpha_{i}}\left(0<\alpha_{i} \leq 1\right)$ is a reflexive and separable Banach space (see [22], Proposition 3.1) for details.

For every $u_{i} \in H_{0}^{\alpha_{i}}$, set

$$
\begin{gathered}
\left\|u_{i}\right\|_{L^{s}}:=\left(\int_{0}^{T}\left|u_{i}(t)\right|^{s} d t\right)^{1 / s}, \quad s \geq 1 \\
\left\|u_{i}\right\|_{\infty}=\max _{t \in[0, T]}\left|u_{i}(t)\right| .
\end{gathered}
$$

Definition 3 [27]. We mean by a weak solution of system (3), any $u=\left(u_{1}, u_{2}, \cdots, u_{n}\right) \in X$ such that for all $v=\left(v_{1}, v_{2}\right.$ $\left., \cdots, v_{n}\right) \in X$, 


$$
\begin{aligned}
& \int_{0}^{T} \sum_{i=1}^{n} a_{i}(t)_{0} D_{t}^{\alpha_{i}} u_{i}(t)_{0} D_{t}^{\alpha_{i}} v_{i}(t) d t \\
& \quad-\lambda \int_{0}^{T} \sum_{i=1}^{n} F_{u_{i}}\left(t, u_{1}(t), u_{2}(t), \cdots, u_{n}(t)\right) v_{i}(t) d t \\
& \quad-\int_{0}^{T} \sum_{i=1}^{n} h_{i}\left(u_{i}\right) v_{i}(t) d t=0 .
\end{aligned}
$$

Lemma 4 [27]. Let $0<\alpha_{i} \leq 1$, for $1 \leq i \leq n$. $\forall u_{i} \in H_{0}^{\alpha_{i}}$, we have

$$
\left\|u_{i}\right\|_{\left(L^{2}\right)} \leq \frac{T^{\alpha_{i}}}{\Gamma\left(\alpha_{i}+1\right)}\left\|{ }_{0} D_{t}^{\alpha_{i}} u_{i}\right\|_{L^{2}}
$$

Moreover,

$$
\left\|u_{i}\right\|_{\infty} \leq \frac{T^{\alpha_{i}}}{\Gamma\left(\alpha_{i}\right) \sqrt{\left(2 \alpha_{i}-1\right)}}\left\|{ }_{0} D_{t}^{\alpha_{i}} u_{i}\right\|_{L^{2}} .
$$

From Lemma 4, we easily observe that

$$
\left\|u_{i}\right\|_{L^{2}} \leq \frac{T^{\alpha_{i}}}{\Gamma\left(\alpha_{i}+1\right) \sqrt{\alpha_{i 0}}}\left(\int_{0}^{T} a_{i}(t)\left|{ }_{0} D_{t}^{\alpha_{i}} u_{i}(t)\right|^{2} d t\right)^{1 / 2}
$$

for $0<\alpha_{i} \leq 1$, and

$$
\left\|u_{i}\right\|_{\infty} \leq \frac{T^{\alpha_{i}-(1 / 2)}}{\Gamma\left(\alpha_{i}\right) \sqrt{a_{i 0}\left(2 \alpha_{i}-1\right)}}\left(\int_{0}^{T} a_{i}(t)\left|{ }_{0} D_{t}^{\alpha_{i}} u_{i}(t)\right|^{2} d t\right)^{1 / 2}
$$

By using (15), the norm of (10) is equivalent to

$$
\left\|u_{i}\right\|_{\alpha_{i}}\left(\int_{0}^{T} a_{i}(t)\left|{ }_{0} D_{t}^{\alpha_{i}} u_{i}(t)\right|^{2} d t\right)^{1 / 2}, \quad \forall u_{i} \in H_{0}^{\alpha_{i}}
$$

Throughout this paper, let $X$ be the Cartesian product of the $n$ spaces $H_{0}^{\alpha_{i}}$ for $1 \leq i \leq n$, i.e., $X=H_{0}^{\alpha_{1}} \times H_{0}^{\alpha_{2}} \times \cdots \times$ $H_{0}^{\alpha_{n}}$; we equip $X$ with the norm defined by

$$
\|u\|=\sum_{i=1}^{n}\left\|u_{i}\right\|_{H_{0}^{\alpha_{i}}}, \quad u=\left(u_{1}, u_{2}, \cdots, u_{n}\right)
$$

where $\left\|u_{i}\right\|_{H_{0}^{\alpha_{i}}}$ is given in (17). We have $X$ compactly embedded in $C([0, T], \mathbb{R})^{n}$.

Theorem 5 [25]. Let $X$ be a reflexive real Banach space and $\Phi: X \longrightarrow \mathbb{R}$ be a coercive, continuously Gâteaux differentiable sequentially weakly lower semicontinuous functional whose Gâteaux derivative admits a continuous inverse on $X^{*}$, bounded on bounded subsets of $X, \Psi: X \longrightarrow \mathbb{R}$ a continuously Gâteaux differentiable functional whose Gâteaux derivative is compact such that

$$
\Phi(0)=\Psi(0)=0
$$

Suppose that $\exists r>0$ and $\bar{x} \in X$, with $r<\Phi(\bar{x})$, satisfying $\left(a_{1}\right) \sup _{\Phi(u) \leq r}(\Psi(u) / r)<(\Phi(\bar{x}) / \Psi(\bar{x}))$.

$\left(a_{2}\right)$ For each $\lambda \in \Lambda_{\lambda} ;=\left(\Phi(\bar{x}) / \Psi(\bar{x}), r / \sup _{\Phi(u) \leq r} \Psi(u)\right)$, the functional $\Phi-\lambda \Psi$ is coercive.

Hence, $\forall \lambda \in \Lambda_{\lambda}$, the functional $\Phi-\lambda \Psi$ has at least three critical points in the space $X$.

\section{Main Results}

In this section, by applying Theorem 5, we examine the existence of multiple solutions for system (3). For any $\sigma>0$, let us define

$$
\pi(\sigma)=\left\{\left(x_{1}, \cdots, x_{n}\right) \in \mathbb{R}^{n}: \frac{1}{2} \sum_{i=1}^{n}\left|x_{i}\right|^{2} \leq \sigma\right\} .
$$

This set will be used in some of our hypotheses with appropriate choices of $\sigma$. For $u=\left(u_{1}, u_{2}, \cdots, u_{n}\right) \in X$, we define

$$
Y(u):=\sum_{i=1}^{n} Y_{i}\left(u_{i}\right)
$$

where $Y_{i}(x)=\int_{0}^{T} H_{i}(x(s)) d s$ and $H_{i}(x)=\int_{0}^{x} h_{i}(z) d z 1 \leq i \leq n$, $\forall t \in[0 ; T]$ and $x \in \mathbb{R}$.

Furthermore, let

$$
\begin{gathered}
k:=\max _{1 \leq i \leq n}\left\{\frac{T^{2 \alpha_{i}-1}}{\left(\Gamma\left(\alpha_{i}\right)\right)^{2} a_{i 0}\left(2 \alpha_{i}-1\right)}\right\}, \\
M:=\min _{1 \leq i \leq n}\left\{1-\frac{L_{i} T^{2 \alpha_{i}}}{\left(\Gamma\left(\alpha_{i}+1\right)\right)^{2} a_{i 0}}\right\}, \\
\tilde{k}:=\max _{1 \leq i \leq n}\left\{1+\frac{L_{i} T^{2 \alpha_{i}}}{\left(\Gamma\left(\alpha_{i}+1\right)\right)^{2} a_{i 0}}\right\} .
\end{gathered}
$$

Theorem 6. Let $1 / 2<\alpha_{i} \leq 1$, for $1 \leq i \leq n$, and suppose that $M>0$ and the conditions (FO) and (F1) are satisfied. Furthermore, assume that $\exists r>0$ and a function $\omega=\left(\omega_{1}, \omega_{2}, \cdots, \omega_{n}\right)$ $\in X$ satisfying

$$
\text { (i) } \sum_{i=1}^{n} \frac{\left\|\omega_{i}\right\|_{\alpha_{i}}^{2}}{2}>\frac{r}{M} \text {, }
$$




$$
\text { (ii) } \begin{aligned}
2 r & \frac{\int_{0}^{T} F\left(t, \omega_{1}, \omega_{2}, \cdots, \omega_{n}\right) d t}{\sum_{i=1}^{n}\left\|\omega_{i}\right\|_{\alpha_{i}}^{2}-2 Y\left(\omega_{1}, \omega_{2}, \cdots, \omega_{n}\right)} \\
& -\int_{0}^{T} \max _{\left(x_{1}, \cdots, x_{n}\right) \in \pi\left(\frac{k r}{M}\right)}, \quad F\left(t, x_{1}, \cdots, x_{n}\right) d t>0,
\end{aligned}
$$

(iii) $\lim _{\left(\left|x_{1}\right|, \cdots,\left|x_{n}\right|\right) \longrightarrow(+\infty, \cdots,+\infty)} \sup \frac{\sup _{t \in[0, T]} F\left(t, x_{1}, \cdots, x_{n}\right)}{\sum_{i=1}^{n}\left|x_{i}\right|^{2} / 2} \leq 0$.

Then, setting

$$
\Lambda=\left(\frac{\sum_{i=1}^{\mathrm{n}}\left(\left\|\omega_{i}\right\|_{\alpha_{i}}^{2} / 2\right)-Y\left(\omega_{1}, \omega_{2}, \cdots, \omega_{n}\right)}{\int_{0}^{T} F\left(t, \omega_{1}(t), \omega_{2}(t), \cdots, \omega_{n}(t)\right) d t}, \frac{r}{\int_{0}^{T} \max _{\left(x_{1}, \cdots, x_{n}\right) \in \pi(M / k r)} F\left(t, x_{1}, \cdots, x_{n}\right) d t}\right) .
$$

$\forall \lambda \in \Lambda$ system (3) admits at least 3 weak solutions in $X$.

Proof. For each $u=\left(u_{1}, u_{2}, \cdots, u_{n}\right) \in X$, we introduce the functionals $\Phi, \Psi: X \longrightarrow \mathbb{R}$ as

$$
\begin{gathered}
\Phi(u)=\sum_{i=1}^{n} \frac{\left\|u_{i}\right\|_{\alpha_{i}}^{2}}{2}-Y(u), \\
\Psi(u)=\int_{0}^{T} F\left(t, u_{1}(t), u_{2}(t), \cdots, u_{n}(t)\right) d t .
\end{gathered}
$$

It is clear that $\Phi$ and $\Psi$ are continuously Gâteaux differentiable functionals whose Gâteaux derivatives at the point $u \in X$ are defined by

$$
\begin{aligned}
\Phi^{\prime}(u)(v)= & \int_{0}^{T} \sum_{i=1}^{n} a_{i}(t)_{0} D_{t}^{\alpha_{i}} u_{i}(t)_{0} D_{t}^{\alpha_{i}} v_{i}(t) d t \\
& -\int_{0}^{T} \sum_{i=1}^{n} h_{i}\left(u_{i}(t)\right) v_{i}(t) d t \\
\Psi^{\prime}(u)(v)= & \int_{0}^{T} \sum_{i=1}^{n} F_{u_{i}}\left(t, u_{1}(t), u_{2}(t), \cdots, u_{n}(t)\right) v_{i}(t) d t
\end{aligned}
$$

for every $v=\left(v_{1}, v_{2}, \cdots, v_{n}\right) \in X$.

We have $\Phi^{\prime}(u), \Psi^{\prime}(u) \in X^{*}$, where $X^{*}$ is the dual space of $X$. And the functional $\Phi$ is sequentially weakly lower semicontinuous and its Gâteaux derivative admits a continuous inverse on $X^{*}$; also $\lim _{\|u\|_{X} \longrightarrow+\infty} \Phi(u)=+\infty$ it is coercive. Now, we show that the functional $\Psi$ is sequentially weakly upper semicontinuous and its derivative $\Psi^{\prime}: X \longrightarrow X^{*}$ is a compact operator. Let $u_{m} \rightarrow u$ in $X$, where $u_{m}(t)=\left(u_{m, 1}(t)\right.$, $\left.u_{m, 2}(t), \cdots, u_{m, n}(t)\right)$; then certainly $u_{m}$ converges uniformly to $u$ on the interval $[0, T]$. Then,

$$
\begin{aligned}
\limsup _{m \longrightarrow+\infty} \Psi\left(u_{m}\right) & \leq \int_{0}^{T} \limsup _{m \longrightarrow+\infty} F\left(t, u_{m, 1}(t), u_{m, 2}(t), \cdots, u_{m, n}(t)\right) d t \\
& =\int_{0}^{T} F\left(t, u_{1}(t), u_{2}(t), \cdots, u_{n}(t)\right) d t=\Psi(u)
\end{aligned}
$$

which gets that $\Psi$ is sequentially weakly upper semicontinuous.

Moreover, we have

$$
\begin{aligned}
& \lim _{m \longrightarrow+\infty} F\left(t, u_{m, 1}(t), u_{m, 2}(t), \cdots, u_{m, n}(t)\right) \\
& \quad=F\left(t, u_{1}(t), u_{2}(t), \cdots, u_{n}(t)\right), \quad \text { for all } t \in[0, T] .
\end{aligned}
$$

Note that $F(t, \cdot, \cdots, \cdot) \in C^{1}\left(\mathbb{R}^{n}\right)$. The Lebesgue control convergence theorem implies that $\Psi_{m}^{\prime}(u) \longrightarrow \Psi^{\prime}(u)$ strongly, hence yielding that $\Psi^{\prime}$ is strongly continuous on $X$. Then, $\Psi^{\prime}: X \longrightarrow X^{*}$ is a compact operator.

We show that required hypothesis $\Phi(\bar{x})>r$ follows from (i) and the definition of $\Phi$ by taking $\bar{x}=\omega$. Indeed, as (5) holds for all $x_{1} ; x_{2} \in \mathbb{R}$ and $h_{1}(0)=\cdots=h_{n}(0)=0$; one has $\left|h_{i}(x)\right|$ $\leq L_{i}|x|, 1 \leq i \leq n$, for any $x \in \mathbb{R}$. It follows from (15) that

$$
\begin{aligned}
\Phi(\omega) & \geq \frac{\sum_{i=1}^{n}\left\|\omega_{i}\right\|_{\alpha_{i}}^{2}}{2}-\left|\int_{0}^{T} \sum_{i=1}^{n} H_{i}\left(\omega_{i}(t)\right) d t\right| \\
& \geq \frac{\sum_{i=1}^{n}\left\|\omega_{i}\right\|_{\alpha_{i}}^{2}}{2}-\sum_{i=1}^{n} \frac{L_{i}}{2} \int_{0}^{T}\left|\omega_{i}(t)\right|^{2} d t \\
& \geq \sum_{i=1}^{n}\left(\frac{1}{2}-\frac{L_{i} T^{2 \alpha_{i}}}{\left(\Gamma\left(\alpha_{i}+1\right)\right)^{2} a_{i 0}}\right)\left\|\omega_{i}\right\|_{\alpha_{i}}^{2} \\
& \geq \frac{M}{2} \sum_{i=1}^{n}\left\|\omega_{i}\right\|_{\alpha_{i}}^{2} .
\end{aligned}
$$


From (16), for every $u_{i} \in H_{0}^{\alpha_{i}}$, we have

$$
\max _{t \in[0, T]}\left|u_{i}(t)\right|^{2} \leq k\left\|u_{i}\right\|_{\alpha_{i}}
$$

for $1 \leq i \leq n$. Hence,

$$
\max _{t \in[0, T]} \sum_{i=1}^{n}\left|u_{i}(t)\right|^{2} \leq k \sum_{i=1}^{n}\left\|u_{i}\right\|_{\alpha_{i}} .
$$

Assume that $u_{0}(t)=(0, \cdots, 0)$ and the supposition $(i)$ deduces that $0<r<\Phi(\omega)$ and they hold $\Phi\left(u_{0}(t)\right)=\Psi\left(u_{0}(t)\right.$ )$=0$ from definitions (25) and (26), which are required assumptions in Theorem 5. Applying relations (16), (17), and (22) gives the following relation:

$$
\begin{aligned}
\Phi^{-1}((-\infty ; r])= & \left\{u=\left(u_{1}, u_{2}, \cdots, u_{n}\right) \in X: \Phi(u) \leq r\right\} \\
= & \left\{u=\left(u_{1}, u_{2}, \cdots, u_{n}\right) \in X: \sum_{i=1}^{n} \frac{\left\|u_{i}\right\|_{\alpha_{i}}^{2}}{2} \leq \frac{r}{M}\right\} \\
\subseteq & \left\{u=\left(u_{1}, u_{2}, \cdots, u_{n}\right) \in X: \sum_{i=1}^{n}\right. \\
& \cdot \frac{\left(\Gamma\left(\alpha_{i}\right)\right)^{2} a_{10}\left(2 \alpha_{i}-1\right)}{\left.2 T^{2 \alpha_{i}-1}\left\|u_{i}\right\|_{\infty}^{2} \leq \frac{r}{M}\right\}} \\
\subseteq & \left\{u=\left(u_{1}, u_{2}, \cdots, u_{n}\right) \in X: \frac{1}{2} \sum_{i=1}^{n}\left|u_{i}(t)\right|^{2} \leq \frac{k r}{M}\right\},
\end{aligned}
$$

which implies that

$$
\begin{aligned}
\sup _{u \in \Phi^{-1}((-\infty ; r])} \Psi(u) & =\sup _{u \in \Phi^{-1}((-\infty ; r])} \int_{0}^{T} F\left(t, u_{1}(t), u_{2}(t), \cdots, u_{n}(t)\right) d t \\
& \leq \int_{0}^{T} \max _{\left(x_{1}, \cdots, x_{n}\right) \in \pi\left(\frac{k r}{M}\right)} F\left(t, x_{1}, \cdots, x_{n}\right) d t .
\end{aligned}
$$

Hence, under the condition (ii), we get the following inequality

$$
\begin{aligned}
\sup _{\frac{u \in \Phi^{-1}((-\infty ; r])}{r} \Psi(u)} & \leq \int_{0}^{T} \max _{\left(x_{1}, \cdots, x_{n}\right) \in \pi\left(\frac{k r}{M}\right)} F\left(t, x_{1}, \cdots, x_{n}\right) d t \\
& <2 r \frac{\int_{0}^{T} F\left(t, \omega_{1}, \omega_{2}, \cdots, \omega_{n}\right) d t}{\sum_{i=1}^{n}\left\|\omega_{i}\right\|_{\alpha_{i}}^{2}-2 Y\left(\omega_{1}, \omega_{2}, \cdots, \omega_{n}\right)} \\
& =r \frac{\int_{0}^{T} F\left(t, \omega_{1}, \omega_{2}, \cdots, \omega_{n}\right) d t}{\sum_{i=1}^{n}\left(\left\|\omega_{i}\right\|_{\alpha_{i}}^{2} / 2\right)-Y\left(\omega_{1}, \omega_{2}, \cdots, \omega_{n}\right)} \\
& =\frac{\Psi(\omega)}{\Phi(\omega)} .
\end{aligned}
$$

Thus, the hypothesis $\left(a_{1}\right)$ of Theorem 5 holds.
On the other hand, fix $0<\varepsilon<(1 / 2 T k \lambda)$. From (iii) into account, there exist constants $\tau_{\varepsilon} \in \mathbb{R}$ such that

$$
F\left(t, x_{1}, \cdots, x_{n}\right) \leq \varepsilon \sum_{i=1}^{n}\left|x_{i}\right|^{2}+\tau_{\varepsilon},
$$

for any $t \in[0, T]$ and $\left(x_{1}, \cdots, x_{n}\right) \in \mathbb{R}^{n}$, by using (36) and (15) yields, it follows that, for each $u \in X$,

$$
\begin{aligned}
\Phi(u)-\lambda \Psi(u) & =\frac{1}{2} \sum_{i=1}^{n}\left\|u_{i}\right\|_{\alpha_{i}}^{2}-\lambda \int_{0}^{T} F\left(t, u_{1}(t), u_{2}(t), \cdots, u_{n}(t)\right) d t \\
& \geq \frac{1}{2} \sum_{i=1}^{n}\left\|u_{i}\right\|_{\alpha_{i}}^{2}-T \lambda k \varepsilon \sum_{i=1}^{n}\left\|u_{i}\right\|_{\alpha_{i}}^{2}-\lambda \tau_{\varepsilon} \\
& \geq\left(\frac{1}{2}-T \lambda k \varepsilon\right) \sum_{i=1}^{n}\left\|u_{i}\right\|_{\alpha_{i}}^{2}-\lambda \tau_{\varepsilon} .
\end{aligned}
$$

And from him,

$$
\lim _{\|u\|_{X} \longrightarrow+\infty} \Phi(u)-\lambda \Psi(u)=+\infty
$$

Moreover, analogous to the case of $\tau_{\varepsilon}>0$, we imply that $\Phi(u)-\lambda \Psi(u) \longrightarrow+\infty$ as $\|u\|_{X} \longrightarrow+\infty$ with $\tau_{\varepsilon} \leq 0$. Then, the hypotheses of Theorem 5 hold, which means that system (3) admits at least 3 weak solutions in $X$, which completes the proof.

Now, we present some notations, before the corollary of Theorem 6.

Put

$$
\begin{aligned}
A_{i}\left(\alpha_{i}\right)= & \frac{16}{T^{2}}\left\{\int_{0}^{T} a_{i}(t) t^{2\left(1-\alpha_{i}\right)} d t+\int_{T / 4}^{T} a_{i}(t)\left(t-\frac{T}{4}\right)^{2\left(1-\alpha_{i}\right)} d t\right. \\
& +\int_{3 T / 4}^{T} a_{i}(t)\left(t-\frac{3 T}{4}\right)^{2\left(1-\alpha_{i}\right)} d t-2 \int_{T / 4}^{T} a_{i}(t) \\
& \cdot\left(t^{2}-\frac{T}{4} t\right)^{1-\alpha_{i}} d t-2 \int_{3 T / 4}^{T} a_{i}(t)\left(t^{2}-\frac{3 T}{4} t\right)^{1-\alpha_{i}} d t \\
& \left.+2 \int_{3 T / 4}^{T} a_{i}(t)\left(t^{2}-T t+\frac{3 T^{2}}{16} t\right)^{1-\alpha_{i}} d t\right\}, \\
\Delta_{1}= & \min _{1 \leq i \leq n}\left\{A_{i}\left(\alpha_{i}\right): \text { for } 1 \leq i \leq n\right\}, \\
\Delta_{2}= & \max _{1 \leq i \leq n}\left\{A_{i}\left(\alpha_{i}\right): \text { for } 1 \leq i \leq n\right\} .
\end{aligned}
$$

Corollary 7. Let $1 / 2<\alpha_{i} \leq 1,1 \leq i \leq n$ and supposition (iii) in Theorem 6 holds. Suppose that $\exists \tau>0$ and $d$ such that $\left(\tau / \Delta_{1}\right.$ $k M n)<d^{2}$, and also

$$
\begin{aligned}
\left(i^{\prime}\right) F\left(t, x_{1}, \cdots, x_{n}\right) \geq & 0, \quad \text { for }\left(t, x_{1}, \cdots, x_{n}\right) \\
\in & \left(\left[0, \frac{T}{4}\right] \cup\left[\frac{3 T}{4}, T\right]\right. \\
& \times[0,+\infty) \times \cdots \times[0,+\infty)),
\end{aligned}
$$




$$
\begin{aligned}
\left(i i^{\prime}\right) & \frac{\int_{0}^{T} \max _{\left(x_{1}, \cdots, x_{n}\right) \in \pi(\tau)} F\left(t, x_{1}, \cdots, x_{n}\right) d t}{\tau M} \\
& <\frac{\int_{T / 4}^{3 T / 4} F\left(t, \Gamma\left(2-\alpha_{1}\right) d, \Gamma\left(2-\alpha_{2}\right) d, \cdots, \Gamma\left(2-\alpha_{n}\right) d\right) d t}{n k \tilde{k} \Delta_{2} d^{2}},
\end{aligned}
$$

$$
\left(i i i^{\prime}\right)_{\left(\left|x_{1}\right|, \cdots,\left|x_{n}\right|\right) \longrightarrow(+\infty, \cdots,+\infty)} \lim _{\sup } \frac{\sup _{t \in[0, T]} F\left(t, x_{1}, \cdots, x_{n}\right)}{\sum_{i=1}^{n}\left|x_{i}\right|^{2} / 2} \leq 0
$$

Then, setting

$$
\lambda \in \Lambda^{\prime}=\left(\frac{n \tilde{k} \Delta_{2} d^{2}}{\int_{T / 4}^{3 T / 4} F\left(t, \Gamma\left(2-\alpha_{1}\right) d, \Gamma\left(2-\alpha_{2}\right) d, \cdots, \Gamma\left(2-\alpha_{n}\right) d\right) d t}, \frac{\tau}{k \int_{0}^{T} \max _{\left(x_{1}, \cdots, x_{n}\right) \in \pi(\tau)} F\left(t, x_{1}, \cdots, x_{n}\right) d t}\right)
$$

Thus, system (3) admits at least three weak solutions in $X$

Proof. Choose

$$
\omega_{i}(t)=\left\{\begin{array}{l}
\frac{4 \Gamma\left(2-\alpha_{i}\right) d}{T} t, \quad t \in\left[0, \frac{T}{4}[\right. \\
\Gamma\left(2-\alpha_{i}\right) d, \quad t \in\left[\frac{T}{4}, \frac{3 T}{4}\right] \\
\left.\left.\frac{4 \Gamma\left(2-\alpha_{i}\right) d}{h T}(T-t), \quad t \in\right] \frac{3 T}{4}, T\right] .
\end{array}\right.
$$

We derive

$$
{ }_{0} D_{t}^{\alpha_{i}} \omega_{i}(t)=\left\{\begin{array}{l}
\frac{4 d}{T} t^{1-\alpha_{i}}, \quad t \in\left[0, \frac{T}{4}[,\right. \\
\frac{4 d}{T}\left(t^{1-\alpha_{i}}-\left(t-\frac{T}{4}\right)^{1-\alpha_{i}}\right), \quad t \in\left[\frac{T}{4}, \frac{3 T}{4}\right], \\
\left.\left.\frac{4 d}{T}\left(t^{1-\alpha_{i}}-\left(t-\frac{T}{4}\right)^{1-\alpha_{i}}-\left(t-\frac{3 T}{4}\right)^{1-\alpha_{i}}\right), \quad t \in\right] \frac{3 T}{4}, T\right] .
\end{array}\right.
$$

Moreover,

$$
\begin{aligned}
& \int_{0}^{T} a_{i}(t)\left|{ }_{0} D_{t}^{\alpha_{i}} \omega_{i}(t)\right|^{2} d t \\
& \quad=\int_{0}^{T / 4}+\int_{T / 4}^{3 T / 4}+\int_{3 T / 4}^{T} a_{i}(t)\left|{ }_{0} D_{t}^{\alpha_{i}} \omega_{i}(t)\right|^{p} d t \\
& \quad=2 A_{i}\left(\alpha_{i}\right) d^{2} .
\end{aligned}
$$

Then, $\omega_{i}(0)=\omega_{i}(T)=0, \omega_{i}(t){ }_{0} D_{t}^{\alpha_{i}} \omega_{i}(t) \in L^{2}[0, T], i=1$, $2, \cdots, n$; hence, $\omega=\left(\omega_{1}, \omega_{2}, \cdots, \omega_{n}\right) \in X$, and we have

$$
\left\|\omega_{i}\right\|_{\alpha_{i}}^{2}=\int_{0}^{T} a_{i}(t)\left|{ }_{0} D_{t}^{\alpha_{i}} \omega_{i}(t)\right|^{2} d t=2 A_{i}\left(\alpha_{i}\right) d^{2} .
$$

By (25), for $1 \leq i \leq n$, imply that

$$
\begin{aligned}
\Phi(\omega) & =\Phi\left(\omega_{1}, \omega_{2}, \cdots, \omega_{n}\right) \\
& =\sum_{i=1}^{n} \frac{\left\|\omega_{i}\right\|_{\alpha_{i}}^{2}}{2}-Y(\omega) \\
& \geq \frac{M}{2} \sum_{i=1}^{n}\left\|\omega_{i}\right\|_{\alpha_{i}}^{2} \\
& =M d^{2} \sum_{i=1}^{n} A\left(\alpha_{i}\right) \\
& \geq n M \Delta_{1} d^{2} .
\end{aligned}
$$

Similar to (30) and (46), we have $\Phi(\omega) \leq n \tilde{k} \Delta_{2} d^{2}$.

Let $r=\tau M / k$. From $\left(\tau / \Delta_{1} k M n\right)<d^{2}$, we have

$\cdot \sum_{i=1}^{n} \frac{\left\|\omega_{i}\right\|_{\alpha_{i}}^{2}}{2} \geq \Phi(\omega) \geq n M \Delta_{1} d^{2}>n M \Delta_{1} \times \frac{\tau}{\Delta_{1} k M n}=\frac{r}{M}$.

Thus, the assumption (ii) of Theorem 6 holds.

$\left(i^{\prime}\right)$ implies that

$$
\begin{aligned}
\Psi(\omega)= & \int_{0}^{T} F\left(t, \omega_{1}, \omega_{2}, \cdots, \omega_{n}\right) d t \\
= & \int_{0}^{T / 4} F\left(t, \omega_{1}, \omega_{2}, \cdots, \omega_{n}\right) d t \\
& +\int_{T / 4}^{3 T / 4} F\left(t, \omega_{1}, \omega_{2}, \cdots, \omega_{n}\right) d t \\
& +\int_{3 T / 4}^{T} F\left(t, \omega_{1}, \omega_{2}, \cdots, \omega_{n}\right) d t \\
\geq & \int_{T / 4}^{3 T / 4} F\left(t, \omega_{1}, \omega_{2}, \cdots, \omega_{n}\right) d t
\end{aligned}
$$


Moreover, by condition $\left(i i^{\prime}\right)$, we have

$$
\begin{aligned}
& \frac{\int_{0}^{T} \max _{\left(x_{1}, \cdots, x_{n}\right) \in \pi(k r / M)} F\left(t, x_{1}, \cdots, x_{n}\right) d t}{r} \\
& \quad=\frac{k \int_{0}^{T} \max _{\left(x_{1}, \cdots, x_{n}\right) \in \pi(k r / M)} F\left(t, x_{1}, \cdots, x_{n}\right) d t}{\tau M} \\
& \quad<\frac{\int_{T / 4}^{3 T / 4} F\left(t, \Gamma\left(2-\alpha_{1}\right) d, \Gamma\left(2-\alpha_{2}\right) d, \cdots, \Gamma\left(2-\alpha_{n}\right) d\right) d t}{n \tilde{k} \Delta_{2} d^{2}} \\
& \quad \leq \frac{\int_{T / 4}^{3 T / 4} F\left(t, \Gamma\left(2-\alpha_{1}\right) d, \Gamma\left(2-\alpha_{2}\right) d, \cdots, \Gamma\left(2-\alpha_{n}\right) d\right) d t}{\Phi(\omega)} \\
& \quad \leq \frac{2 \int_{0}^{T} F\left(t, \omega_{1}, \omega_{2}, \cdots, \omega_{n}\right) d t}{\sum_{i=1}^{n}\left\|\omega_{i}\right\|_{\alpha_{i}}^{2}-2 Y\left(\omega_{1}, \omega, \cdots, \omega_{n}\right)} .
\end{aligned}
$$

Hence, the supposition (ii) of Theorem 6 is verified.

Moreover, the supposition (iii) of Theorem 6 holds under ( iii $^{\prime}$ ) from $\Lambda^{\prime} \subseteq \Lambda$. Theorem 6 is successfully employed to ensure the existence of at least 3 weak solutions for system (3). This completes of the proof.

\section{Examples}

In this section, we propose two practical examples of Theorem 6.

Example $\quad 1 . \quad$ Let $\quad \alpha_{1}=0.7, \alpha_{2}=0.65, \alpha_{3}=0.6$, $a_{1}(t)=1+t^{2}, a_{2}(t)=0.5+t, a_{3}(t)=1+t, T=1$. Then, sys- tem (3) gets the following form:

$$
\left\{\begin{array}{c}
{ }_{t} D_{1}^{0.7}\left(\left(1+t^{2}\right){ }_{0} D_{t}^{0.7} u_{1}(t)\right)=\lambda F_{u_{1}}\left(t, u_{1}(t), u_{2}(t), u_{3}(t)\right)+h_{1}\left(u_{1}\right), \quad t \in[0,1], \\
\left.{ }_{t} D_{1}^{0.65}((0.5+t))_{0} D_{t}^{0.65} u_{2}(t)\right)=\lambda F_{u_{2}}\left(t, u_{1}(t), u_{2}(t), u_{3}(t)\right)+h_{2}\left(u_{2}\right), \quad t \in[0,1], \\
{ }_{t} D_{1}^{0.6}\left((1+t)_{0} D_{t}^{0.6} u_{3}(t)\right)=\lambda F_{u_{3}}\left(t, u_{1}(t), u_{2}(t), u_{3}(t)\right)+h_{3}\left(u_{3}\right), \quad t \in[0,1], \\
u_{1}(0)=u_{1}(1)=0, u_{2}(0)=u_{2}(1)=0, u_{3}(0)=u_{3}(1)=0,
\end{array}\right.
$$

where $h_{1}\left(u_{1}\right)=1 / 4 \sin u_{1}, h_{2}\left(u_{2}\right)=u_{2} / 2$, and $h_{3}\left(u_{3}\right)=1 / 20$ $\arctan u_{3}$.

Furthermore, $\forall\left(t ; x_{1}, x_{2}, x_{3}\right) \in[0 ; 1] \times R^{3}$; put

$$
F\left(t, x_{1}(t), x_{2}(t), x_{3}(t)\right)=\left(1+t^{2}\right) G\left(x_{1}, x_{2}, x_{3}\right),
$$

where

$$
\begin{aligned}
& G\left(x_{1}, x_{2}, x_{3}\right) \\
& \quad=\left\{\begin{array}{l}
\left(x_{1}^{2}+x_{2}^{2}+x_{3}^{2}\right)^{2}, x_{1}^{2}+x_{2}^{2}+x_{3}^{2} \leq 1, \\
10\left(x_{1}^{2}+x_{2}^{2}+x_{3}^{2}\right)^{\frac{1}{2}}-9\left(x_{1}^{2}+x_{2}^{2}+x_{3}^{2}\right)^{1 / 3}, x_{1}^{2}+x_{2}^{2}+x_{3}^{2}>1 .
\end{array}\right.
\end{aligned}
$$

Obviously $h_{1}, h_{1}, h_{3} \longrightarrow \mathbb{R}$ are three Lipschitz continuous functions with Lipschitz constants $L_{1}=1 / 4, L_{2}=1 / 2, L_{3}$ $=1 / 20$ and $h_{1}(0)=h_{2}(0)=h_{3}(0)=0$. Clearly, $F(t, 0,0,0)=$ $0, \forall t \in[0,1]$, by the direct calculation, we have $a_{10}=1, a_{20}$ $=1$, and $a_{30}=0.5$

$$
\begin{gathered}
k=\max \left\{\frac{1}{(\Gamma(0.7))^{2}(2 \times 0.7-1)}, \frac{1}{(\Gamma(0.65))^{2}(2 \times 0.65-1)}, \frac{1}{(\Gamma(0.6))^{2} \times 0.5(2 \times 0.6-1)}\right\} \approx 4.509191 \\
M=\min \left\{1-\frac{L_{1}}{(\Gamma(0.7)+1)^{2}}, 1-\frac{L_{2}}{(\Gamma(0.65)+1)^{2}}, 1-\frac{L_{3}}{(\Gamma(0.6)+1)^{2} \times 0.5}\right\} \approx 0.912084
\end{gathered}
$$

Taking

$$
\begin{aligned}
\omega_{1}(t) & =\Gamma(1.3) t(1-t), \omega_{2}(t) \\
& =\Gamma(1.35) t(1-t), \omega_{3}(t) \\
& =\Gamma(1.4) t(1-t) \\
{ }_{0} D_{t}^{0.7} \omega_{1}(t) & =t^{0.3}-\frac{2 \Gamma(1.3)}{\Gamma(2.3)} t^{1.3}, \\
{ }_{0} D_{t}^{0.65} \omega_{2}(t) & =t^{0.35}-\frac{2 \Gamma(1.35)}{\Gamma(2.35)} t^{1.35}, \\
{ }_{0} D_{t}^{0.6} \omega_{3}(t) & =t^{0.4}-\frac{2 \Gamma(1.4)}{\Gamma(2.4)} t^{1.4} .
\end{aligned}
$$

By a simple calculation, we obtain

$$
\begin{aligned}
\left\|\omega_{1}(t)\right\|_{0.7}^{2} & \approx 0.130566,\left\|\omega_{2}(t)\right\|_{0.65}^{2} \\
& \approx 0.078559,\left\|\omega_{3}(t)\right\|_{0.6}^{2} \\
& \approx 0.102638
\end{aligned}
$$

Select $r=1 \times 10^{-3}$, we find

$$
\begin{gathered}
\left\|\omega_{1}(t)\right\|_{0.7}^{2}+\left\|\omega_{2}(t)\right\|_{0.65}^{2}+\left\|\omega_{3}(t)\right\|_{0.6}^{2} \\
\approx 0.311763>\frac{2 r}{M} \approx 0.002192 .
\end{gathered}
$$


We deduce that the supposition (i) holds, and

$$
\begin{aligned}
& \frac{\int_{0}^{1} \max _{\left(x_{1}, x_{2}, x_{3}\right) \in \pi(k r / M)} F\left(t, x_{1}, x_{2}, x_{3}\right) d t}{r} \\
& \quad=\frac{16 k^{2} r}{3 M^{2}} \approx 0.130355 \\
& \quad<\frac{2 \int_{0}^{1} F\left(t, \omega_{1}, \omega_{2}, \omega_{3}\right) d t}{\left(\left\|\omega_{1}(t)\right\|_{0.7}^{2}+\left\|\omega_{2}(t)\right\|_{0.65}^{2}+\left\|\omega_{3}(t)\right\|_{0.6}^{2}\right)-Y\left(\omega_{1}, \omega_{2}, \omega_{3}\right)} \\
& \quad \approx 0.365517,
\end{aligned}
$$

$$
\lim _{\left(\left|x_{1}\right|,\left|x_{2}\right|,\left|x_{3}\right|\right) \longrightarrow(+\infty,+\infty,+\infty)} \sup \frac{\sup _{t \in[0, T]} F\left(t, x_{1}, x_{2}, x_{3}\right)}{\left(\left|x_{1}\right|^{2} / 2\right)+\left(\left|x_{2}\right|^{2} / 2\right)+\left(\left|x_{3}\right|^{2} / 2\right)}=0 .
$$

Then, suppositions (ii) and (iii) are verified. Hence, in view of Theorem 6 for every $\lambda \in] 2.7359,7.6714[$, system (50) has at least 3 weak solutions in the space $X=H_{0}^{0.7} \times H_{0}^{0.65} \times H_{0}^{0.6}$.

Example 2. Let $\alpha_{1}=0.65, \alpha_{2}=0.75, \alpha_{3}=0.85, \alpha_{4}=0.95, a_{1}(t$ )$=1+t^{3}, a_{2}(t)=1+t^{2}, a_{3}(t)=0.5+t, a_{4}(t)=1+t, T=1$.

Hence, system (3) gives

$$
\left\{\begin{array}{l}
{ }_{t} D_{1}^{0.65}\left(\left(1+t^{3}\right){ }_{0} D_{t}^{0.65} u_{1}(t)\right)=\lambda F_{u_{1}}\left(t, u_{1}(t), u_{2}(t), u_{3}(t), u_{4}(t)\right)+h_{1}\left(u_{1}\right), \quad t \in[0,1], \\
{ }_{t} D_{1}^{0.75}\left(\left(1+t^{2}\right)_{0} D_{t}^{0.75} u_{2}(t)\right)=\lambda F_{u_{2}}\left(t, u_{1}(t), u_{2}(t), u_{3}(t), u_{4}(t)\right)+h_{2}\left(u_{2}\right), \quad t \in[0,1], \\
{ }_{t} D_{1}^{0.85}\left((0.5+t){ }_{0} D_{t}^{0.85} u_{3}(t)\right)=\lambda F_{u_{3}}\left(t, u_{1}(t), u_{2}(t), u_{3}(t), u_{4}(t)\right)+h_{3}\left(u_{3}\right), \quad t \in[0,1], \\
{ }_{t} D_{1}^{0.95}\left((1+t){ }_{0} D_{t}^{0.95} u_{4}(t)\right)=\lambda F_{u_{4}}\left(t, u_{1}(t), u_{2}(t), u_{3}(t), u_{4}(t)\right)+h_{4}\left(u_{4}\right), \quad t \in[0,1], \\
u_{1}(0)=u_{1}(1)=0, u_{2}(0)=u_{2}(1)=0, u_{3}(0)=u_{3}(1)=0, u_{4}(0)=u_{4}(1)=0 .
\end{array}\right.
$$

Taking

$$
\begin{aligned}
\omega_{1}(t) & =\Gamma(1.35) t(1-t), \omega_{2}(t) \\
& =\Gamma(1.25) t(1-t), \omega_{3}(t) \\
& =\Gamma(1.15) t(1-t), \omega_{4}(t) \\
& =\Gamma(1.05) t(1-t) .
\end{aligned}
$$

where

$$
G\left(x_{1}, x_{2}, x_{3}, x_{4}\right)=\left\{\begin{array}{l}
\left(x_{1}^{2}+x_{2}^{2}+x_{3}^{2}+x_{4}^{2}\right)^{2}, x_{1}^{2}+x_{2}^{2}+x_{3}^{2}+x_{4}^{2} \leq 1 \\
10\left(x_{1}^{2}+x_{2}^{2}+x_{3}^{2}+x_{4}^{2}\right)^{\frac{1}{2}}-9\left(x_{1}^{2}+x_{2}^{2}+x_{3}^{2}+x_{4}^{2}\right)^{\frac{1}{3}}, x_{1}^{2}+x_{2}^{2}+x_{3}^{2}+x_{4}^{2}>1 .
\end{array}\right.
$$

Obviously $h_{1}, h_{1}, h_{3}, h_{4} \longrightarrow \mathbb{R}$ are three Lipschitz continuous functions, $h_{1}\left(u_{1}\right)=1 / 4 \sin u_{1}, h_{2}\left(u_{2}\right)=u_{2} / 20$ and $h_{3}\left(u_{3}\right.$ )$=1 / 100 \arctan u_{3}, h_{4}\left(u_{4}\right)=1 / 10 \ln \left(u_{4}+1\right)$ for all $u_{1}, u_{2}$, $u_{3}, u_{4} \in \mathbb{R}$ with Lipschitz constants $L_{1}=1 / 4, L_{2}=1 / 20, L_{3}=$ $1 / 100, L_{4}=1 / 10 \quad$ and $\quad h_{1}(0)=h_{2}(0)=h_{3}(0)=h_{4}(0)=0$. Clearly, $F(t, 0,0,0,0)=0$ for any $t \in[0,1], a_{10}=1, a_{20}=0.5$, $a_{30}=1$, and $a_{40}=1$

The direct calculation, gives

$$
k=\max \left\{\begin{array}{c}
\frac{1}{(\Gamma(0.65))^{2}(2 \times 0.65-1)}, \frac{1}{(\Gamma(0.75))^{2} \times 0.5(2 \times 0.75-1)} \\
, \frac{1}{(\Gamma(0.85))^{2}(2 \times 0.85-1)}, \frac{1}{(\Gamma(0.95))^{2}(2 \times 0.95-1)}
\end{array}\right\}
$$$$
\approx 2.663742 \text {, }
$$

Moreover, for all $\left(t ; x_{1}, x_{2}, x_{3}, x_{4}\right) \in[0 ; 1] \times R^{4}$, put

$$
F\left(t, x_{1}(t), x_{2}(t), x_{3}(t), x_{4}(t)\right)=\left(1+t^{2}\right) G\left(x_{1}, x_{2}, x_{3}, x_{4}\right),
$$

$$
\begin{aligned}
M & =\min \left\{\begin{array}{c}
1-\frac{L_{1}}{(\Gamma(0.65)+1)^{2}}, 1-\frac{L_{2}}{(\Gamma(0.75)+1)^{2} \times 0.5} \\
, 1-\frac{L_{3}}{(\Gamma(0.85)+1)^{2}}, 1-\frac{L_{4}}{(\Gamma(0.95)+1)^{2}}
\end{array}\right\} \\
& \approx 0.956042,
\end{aligned}
$$

$$
\begin{aligned}
& { }_{0} D_{t}^{0.65} \omega_{1}(t)=t^{0.35}-\frac{2 \Gamma(1.35)}{\Gamma(2.35)} t^{1.35}, \\
& { }_{0} D_{t}^{0.75} \omega_{2}(t)=t^{0.25}-\frac{2 \Gamma(1.25)}{\Gamma(2.25)} t^{1.25}, \\
& { }_{0} D_{t}^{0.85} \omega_{3}(t)=t^{0.15}-\frac{2 \Gamma(1.15)}{\Gamma(2.15)} t^{1.15}
\end{aligned}
$$




$$
{ }_{0} D_{t}^{0.95} \omega_{3}(t)=t^{0.05}-\frac{2 \Gamma(1.05)}{\Gamma(2.05)} t^{1.05}
$$

So that

$$
\begin{aligned}
\left\|\omega_{1}(t)\right\|_{0.65}^{2} & \approx 0.104555,\left\|\omega_{2}(t)\right\|_{0.75}^{2} \\
& \approx 0.158153,\left\|\omega_{3}(t)\right\|_{0.85}^{2} \\
& \approx 0.170894,\left\|\omega_{4}(t)\right\|_{0.95}^{2} \\
& \approx 0.397611 .
\end{aligned}
$$

Select $r=1 \times 10^{-3}$; we find

$$
\begin{aligned}
& \left\|\omega_{1}(t)\right\|_{0.65}^{2}+\left\|\omega_{2}(t)\right\|_{0.75}^{2} \\
& \quad+\left\|\omega_{3}(t)\right\|_{0.85}^{2}+\left\|\omega_{4}(t)\right\|_{0.95}^{2} \\
& \approx 0.831213>\frac{2 r}{M} \approx 0.002092 .
\end{aligned}
$$

We deduce that the supposition (i) holds, and

$$
\begin{aligned}
& \frac{\int_{0}^{1} \max _{\left(x_{1}, x_{2}, x_{3}, x_{4}\right) \in \pi(k r / M)} F\left(t, x_{1}, x_{2}, x_{3}, x_{4}\right) d t}{r}=\frac{16 k^{2} r}{3 M^{2}} \approx 0.041403 \\
& <\frac{2 \int_{0}^{1} F\left(t, \omega_{1}, \omega_{2}, \omega_{3}, \omega_{4}\right) d t}{\left(\left\|\omega_{1}(t)\right\|_{0.65}^{2}+\left\|\omega_{2}(t)\right\|_{0.75}^{2}+\left\|\omega_{3}(t)\right\|_{0.85}^{2}+\left\|\omega_{4}(t)\right\|_{0.95}^{2}\right)-Y\left(\omega_{1}, \omega_{2}, \omega_{3}, \omega_{4}\right)} \approx 0.135277, \\
& \quad \sup _{t \in[0, T]} F\left(t, x_{1}, x_{2}, x_{3}, x_{4}\right) \\
& \quad \lim _{\left(\left|x_{1}\right|,\left|x_{2}\right|,\left|x_{3}\right|,\left|x_{4}\right|\right) \longrightarrow(+\infty,+\infty,+\infty,+\infty)} \sup \frac{\left.x^{2}\right)}{\left(\left|x_{1}\right|^{2} / 2\right)+\left(\left|x_{2}\right|^{2} / 2\right)+\left(\left|x_{3}\right|^{2} / 2\right)+\left(\left|x_{4}\right|^{2} / 2\right)}=0 .
\end{aligned}
$$

Then, suppositions (ii) and (iii) are verified. Hence, in view of Theorem 6 for every $\lambda \in] 7.3922,24.1528[$, system (58) has at least 3 weak solutions in the space $X=H_{0}^{0.65} \times$ $H_{0}^{0.75} \times H_{0}^{0.85} \times H_{0}^{0.95}$.

\section{Conclusion}

In this work, at least 3 weak solutions were obtained for a new class of nonlinear fractional BVPs using a critical three-point theorem due to Bonano and Marano. Some appropriate function spaces and variational frameworks were successfully created for system (3). Finally, we suggested two practical examples of Theorem 6 with a special case discussion $\mathbb{R}^{3}$. As for case $\mathbb{R}^{4}$, it was discussed. This makes our results prominent and distinct than previous ones. In the next work, we extend our recent work to the coupled system for this important problem. Also some numerical examples will be given in order to ensure the theory study by using some famous algorithms which are presented in $([28,29])$.

\section{Data Availability}

No data were used to support the study.

\section{Conflicts of Interest}

This work does not have any conflicts of interest.

\section{Acknowledgments}

The sixth author extend their appreciation to the Deanship of Scientific Research at King Khalid University for funding this work through Research Group Project under Grant No. R.G.P-2/1/42.

\section{References}

[1] H. M. Baskonus, "Complex surfaces to the fractional $(2+1)$ dimensional Boussinesq dynamical model with the local Mderivative," The European Physical Journal Plus, vol. 134, no. 7, p. 322, 2019.

[2] G. Bonanno and S. Marano, "On the structure of the critical set of non-differentiable functions with a weak compactness condition," Applicable Analysis, vol. 89, no. 1, pp. 1-10, 2010.

[3] G. W. C. Y. Ma, B. Shiri, and D. Baleanu, "New fractional signal smoothing equations with short memory and variable order," Nonlinear Analysis, vol. 218, p. 164507, 2020.

[4] H. E. Dadkhah, B. Shiri, and D. Baleanu, "Visco-elastic dampers in structural buildings and numerical solution with spline collocation methods," Journal of Applied Mathematics and Computing, vol. 63, no. 1-2, pp. 29-57, 2020.

[5] B. S. E. D. Khiabani, H. Ghaffarzadeh, and J. Katebi, "Spline collocation methods for seismic analysis of multiple degree of freedom systems with visco-elastic dampers using fractional models," Journal of Vibration and Control, vol. 26, no. 17-18, pp. 1445-1462, 2020.

[6] F. Jiao and Y. Zhou, "Existence results for fractional boundary value problem via critical point theory," International Journal of Bifurcation and Chaos, vol. 22, no. 4, article 1250086, 2012. 
[7] W. Gao, P. Veeresha, D. G. Prakasha, H. M. Baskonus, and G. Ye, "A powerful approach for fractional Drinfeld-SokolovWilson equation with Mittag-Leffler law," Alexandria Engineering Journal, vol. 58, no. 4, pp. 1301-1311, 2019.

[8] W. Gao, H. F. Ismael, S. A. Mohammed, H. M. Baskonus, and H. Bulut, "Complex and real optical Soliton properties of the paraxial non-linear Schrödinger equation in Kerr media with M-fractional," Frontiers of Physics, vol. 7, pp. 1-8, 2019.

[9] W. Gao, G. Yel, H. M. Baskonus, and C. Cattani, "Complex solitons in the conformable $(2+1)$-dimensional AblowitzKaup-Newell-Segur equation," AIMS Mathematics, vol. 5, no. 1, pp. 507-521, 2020.

[10] G. Wei, P. Veeresha, D. G. Prakasha, H. M. Baskonus, and G. Yel, "New approach for the model describing the deathly disease in pregnant women using Mittag-Leffler function," Solitons and Fractals, vol. 134, p. 109696, 2020.

[11] M. J. H. J. Ul Rahman, D. Lu, and M. Ramzan, "He-Elzaki method for spatial diffusion of biological population," Fractals, vol. 27, no. 5, article 1950069, 2019.

[12] M. Suleman, D. Lu, J. H. He, U. Farooq, Y. S. Hui, and J. U. Rahman, "Numerical investigation of fractional HIV model using Elzaki projected differential transform method," Fractals, vol. 26, no. 5, article 1850062, 2018.

[13] I. Podlubny, Fractional Differential Equations, Academic Press, San Diego, 1999.

[14] B. Ricceri, "A three critical points theorem revisited," Nonlinear Analysis, vol. 70, no. 9, pp. 3084-3089, 2009.

[15] G. W. B. Shiri and D. Baleanu, "Collocation methods for terminal value problems of temperedfractional differential equations," Applied Numerical Mathematics, vol. 156, pp. 385-395, 2020.

[16] S. Rashid, F. Jarad, M. A. Noor, H. Kalsoom, and Y. M. Chu, "Inequalities by means of generalized proportional fractional integral operators with respect to another function," Mathematics, vol. 7, no. 12, pp. 1-18, 2019.

[17] S. Rashid, F. Jarad, H. Kalsoom, and Y. M. Chu, "On PólyaSzegö and Čebyšev type inequalities via generalized kfractional integrals," Advances in Difference Equations, vol. 2020, no. 1, 2020.

[18] S. Rashid, F. Jarad, and Y. M. Chu, "A note on reverse Minkowski inequality via generalized proportional fractional integral operator with respect to another function," Mathematical Problems in Engineering, vol. 2020, Article ID 7630260, 12 pages, 2020.

[19] E. Shivanian, "Existence of at least three distinct weak solutions for a class of nonlinear system of fractional differential equations," Numerical Functional Analysis and Optimization, vol. 41 , no. 10, pp. 1228-1245, 2020.

[20] H. Y. Zhao and Q. Zhang, "Infinitely many solutions for fractional differential system via variational method," Journal of Applied Mathematics and Computing, vol. 50, no. 1-2, pp. 589-609, 2016.

[21] A. M. V. Kokilashvili and M. Ragusa, "Weighted extrapolation in grand Morrey spaces and applications to partial differential equations," Rendiconti Lincei-Matematica e Applicazioni, vol. 30, no. 1, pp. 67-92, 2019.

[22] H. Y. Zhao and B. Y. Qin, "Multiple solutions for a coupled system of nonlinear fractional differen-tial equations via variational methods," Applied Mathematics and Computation, vol. 257, pp. 417-427, 2015.
[23] K. Oldham and J. Spanier, The Fractional Calculus Theory and Applications of Differentiationand Integration to Arbitrary Order, Academic Press, New York, NY, 1974.

[24] R. Metzler and J. Klafter, "The restaurant at the end of the random walk: recent developments in the description of anomalous transport by fractional dynamics," Journal of Physics A: Mathematical and General, vol. 37, no. 31, pp. R161-R208, 2004.

[25] M. Ragusa, "Commutators of fractional integral operators on vanishing-Morrey spaces," Journalof Global Optimization, vol. 40, no. 1-3, pp. 361-368, 2008.

[26] B. Shiri and D. Baleanu, "Numerical solution of some fractional dynamical systems in medicineinvolving non-singular kernel with vector order," Results in Nonlinear Analysis, vol. 2, no. 4, pp. 160-168, 2019.

[27] B. Karaman, "Analytical investigation for modified RiemannLouville fractional equal-with equation types based on (G '/G) expansion technique," Miskolc Mathematical Notes, vol. 21, no. 1, pp. 219-227, 2020.

[28] S. Boulaaras and M. Haiour, "The finite element approximation of evolutionary Hamilton-Jacobi-Bellman equations with nonlinear source terms," Indagationes Mathematicae, vol. 24, no. 1, pp. 161-173, 2013.

[29] S. Boulaaras and M. Haiour, “_ $\mathrm{L}_{-}{ }^{\infty}$-asymptotic behavior for a finite element approximation in parabolic quasi-variational inequalities related to impulse control problem," Applied Mathematics and Computation, vol. 217, no. 14, pp. 64436450, 2011. 